\title{
Avaliação comparativa do efeito dos colírios de apraclonidina e latanoprost na profilaxia da hipertensão ocular pós capsulotomia com YAG Laser em pacientes não glaucomatosos*
}

\author{
Profilatic use of apraclonidine and latanoprost to prevent intraocular pressure \\ elevation following $\mathrm{Nd}: \mathrm{YAG}$ laser posterior capsulotomy
}

Juliane de Freitas S. Paranhos ${ }^{(1)}$

Augusto Paranhos Jr. ${ }^{(2)}$

João Jorge Nassaralha Jr. ${ }^{(3)}$

\section{RESUMO}

Objetivo: Comparar a eficácia do latanoprost $0,005 \%$ e da apraclonidina $1 \%$ na prevenção da hipertensão ocular póscapsulotomia posterior com YAG laser, em pacientes nãoglaucomatosos.

Método: Trinta e seis pacientes (36 olhos) incluídos foram divididos em três grupos que usaram latanoprost $0,005 \%$, apraclonidina $1 \%$ ou placebo. Foram realizadas medidas da pressão ocular antes da instilação de qualquer colírio, 30 minutos após a dilatação, 1 hora e 4 horas após o YAG laser.

Resultados: Não houve diferença significativa entre os 3 grupos em relação à idade, pressão ocular inicial e pós-dilatação, assim como em quantidade de energia utilizada. Na primeira hora, tanto para a diferença absoluta quanto para o percentual de alteração da pressão intra-ocular, o grupo da apraclonidina apresentou redução estatisticamente maior em relação ao grupo controle $(\mathbf{p}<0,05)$. 0 grupo do latanoprost não diferiu estatisticamente dos dois outros $\operatorname{grupos}(p>0,05)$. Na $4^{\text {a. }}$ hora não houve diferença significativa entre os grupos $(p>0,05)$. As medidas de pressão nos 4 intervalos não apresentaram variação estatisticamente significativa $(p>0,05)$. A quantidade de energia utilizada não mostrou correlação significativa com o aumento da $P o(p>0,05)$.

Conclusão: A apraclonidina 1\% apresentou maior eficácia na profilaxia da hipertensão após capsulotomia posterior com YAG laser quando comparada com latanoprost $0,005 \%$.

Palavras-chave: Glaucoma: capsulotomia, hipertensão ocular, apraclonidina, latanoprost prevenção.

\section{INTRODUÇÃ̃O}

Apresentado no XIII Congresso de Prevenção da Cegueira e Reabilitação Visual- 7 a 10/9/98 - Rio de Janeiro - RJ - Brasil

Médica Residente do Instituto de Olhos de Goiânia

Mestre em Oftalmologia pela Escola Paulista de Medicina.

(3) Setor de Retina e Vítreo do Instituto de Olhos de Goiânia, Setor de Ecografia Ocular do Instituto de Olhos de Goiânia, Coordenador da Residência Médica do Instituto de Olhos de Goiânia.

Endereço para correspondência: Instituto de Olhos de Goiânia. Rua 9-B, 48 - Setor Oeste - Goiânia (GO) CEP 74110-120. 
aumento da pressão intra-ocular (Po) após este procedimento são os glaucomatosos, afácicos e os pacientes com alta miopia ${ }^{5,15}$.

Várias drogas já foram estudadas para profilaxia da hipertensão ocular pós-laser, como acetazolamida, pilocarpina, timolol, mas a apraclonidina tem sido a droga mais apontada para este fim 2,6-8,9-13. O latanoprost é uma droga nova e, segundo Philip e col., mostrou agir sinergicamente quando associada a vários tipos de drogas anti-glaucomatosas com diferentes mecanismos de ação: redução da produção de humor aquoso, aumento do fluxo úveo-escleral e drogas com formas de ação mista ${ }^{14}$.

O objetivo deste trabalho é comparar a eficácia da prevenção da hipertensão ocular pós-YAG laser para capsulotomia posterior usando-se latanoprost $0,005 \%$ ou apraclonidina $1 \%$.

\section{PACIENTES E MÉTODOS}

O trabalho foi submetido e aprovado pelo Comitê de Ética do Instituto de Olhos de Goiânia.

Critérios de inclusão: Pacientes pseudo-fácicos com opacificação capsular posterior operados há, no mínimo, 6 meses. Ausência de glaucoma, alta miopia (acima de 6 D), gravidez e afecções que pudessem interferir na medida da pressão ocular.

O estudo é um experimento clínico prospectivo, duplo mascarado, aleatorizado (sorteio individual).

Dividiu-se os pacientes em três grupos:

Grupo A: Recebeu 1 gota de latanoprost 0,005\% (Xala$\tan ^{\circledR}$ - Pharmacia \& Upjon) 1 hora antes e imediatamente após a cirurgia.

Grupo B: Recebeu 1 gota de apraclonidina 1,0\% (Iopidine $^{\circledR}$ - Alcon) 1 hora antes e imediatamente após a cirurgia.

Grupo C: Recebeu 1 gota de placebo (álcool polivinílico - Lacril $^{\circledR}$ - Allergan) 1 hora antes e imediatamente após a cirurgia.

Os médicos que realizaram a capsulotomia e as medidas da Po assim como os pacientes não tiveram acesso ao tipo de colírio que foi aplicado.

Antes da cirurgia todos os pacientes tiveram suas pupilas dilatadas com 1 gota de tropicamida $1 \%$ e fenilefrina $10 \%$ (cloridrato de fenilefrina, $1 \mathrm{mg}$ )

A medida da Po foi obtida usando-se um tonômetro de aplanação de Goldmann. Quatro medidas foram tomadas. Antes de cada medida foi instilada 1 gota de Anestésico ${ }^{\circledR}$ (cloridrato de tetracaína, $10 \mathrm{mg}$, Frumtost). A primeira medida foi tomada antes da instilação dos colírios tropicamida e fenilefrina, a segunda 30 minutos após a midríase, a terceira e a quarta, 1 hora e 4 horas após o laser, respectivamente. Estas medidas foram tomadas sempre nos mesmos horários. Após a midríase aplicou-se o colírio hipotensor e o laser foi realizado 1 hora depois. Imediatamente antes da capsulotomia instilou-se 1 gota de colírio Anestésico ${ }^{\circledR}$.

A capsulotomia posterior com YAG Laser foi realizada por um dos autores (JJNJr). Iniciava-se com 3,0 mJ por tiro e a energia era aumentada ou diminuída o quanto fosse necessário.

Foi considerada hipertensão ocular aumentos de Po acima de $8 \mathrm{mmHg}$. Caso o paciente tivesse um aumento clinicamente significativo da pressão intra-ocular após o laser, seria administrada medicação para controle e far-se-ia descrição do caso.

A energia total do laser utilizada foi calculada multiplicando-se a quantidade de energia de cada disparo pelo número de disparos.

A Po foi analisada em seu valor absoluto, porcentagem de variação e diferença entre os períodos e a Po inicial $(\Delta \mathrm{Po})$. A opção de testes paramétricos ou não paramétricos foi feita baseada na normalidade das distribuições, avaliada por meio do teste de Kolmogorov-Smirnov. A comparação entre os grupos quanto às variáveis: idade, energia utilizada, Po em cada intervalo, $\Delta \mathrm{Po}$ e da porcentagem de variação de Po, foi feita por meio de Análise de Variância (ANOVA) "one way" com teste de Bofferroni para comparações múltiplas. Para a comparação dos valores de Po em cada grupo, nos quatro intervalos foi utilizado o teste de ANOVA por postos de Friedman. O teste de correlação de Spearman foi utilizado para avaliar a relação entre o total de energia e aumento da Po. Adotou-se como nível de significância $\mathrm{p}<0,05$.

Este estudo obedeceu às normas da declaração de Helsinki para experimentação clínica.

\section{RESULTADOS}

Trinta e seis pacientes foram incluídos neste estudo, sendo 18 homens e 18 mulheres. Estes pacientes foram divididos em 03 grupos. Grupo A com 14 pacientes e grupos B e C com 11 pacientes cada. A estatística descritiva das medidas de Po assim como as diferenças de cada período em relação a Po inicial estão listados na Tabela 1 .

A idade variou de 7 a 88 anos com média de 58,8 anos $( \pm$ $21,7)$. Não houve diferença estatisticamente significativa entre as idades dos 3 grupos $(\mathrm{p}=0,6571)$.

Não houve diferença estatisticamente significativa entre as Po inicial $(p=0,351)$, pós ciclo $(p=0,6373)$, nem com a quantidade de energia utilizada $(\mathrm{p}=0,4824)$. Portanto, conclui-se pela comparabilidade dos grupos.

Na primeira hora após o procedimento, não foi encontrada diferença estatisticamente significativa entre as pressões nos três grupos $(p=0,2015)$, esta diferença também não foi encontrada na quarta hora $(\mathrm{p}=0,0863)$.

A maior mediana de Po, $12 \mathrm{mmHg}$, e a maior Po individual, $36 \mathrm{mmHg}$, foram observadas no grupo controle. Houveram 2 casos $(18,2 \%)$ de aumento da $\mathrm{Po} \geq 8 \mathrm{mmHg}$ no grupo controle e nenhum caso nos grupos tratados com latanoprost e apraclonidina. Um paciente teve aumento de $16 \mathrm{mmHg}$ na primeira hora aumentando para $18 \mathrm{mmhg}$ na quarta hora a Po inicial neste paciente foi $18 \mathrm{mmHg}$, o outro paciente teve aumento de $4 \mathrm{mmHg}$ na primeira hora aumentando para 8 $\mathrm{mmHg}$, sua Po inicial era de $8 \mathrm{mmHg}$. Para o primeiro paciente citado foi receitado Betoptic $\mathrm{S}^{\circledR}$ (Cloridrato de betaxolol $0,25 \%$, Alcon) de 12 em 12 horas por 5 dias, para o outro paciente não foi receitado nenhuma medicação anti-glaucomatosa tendo sido feito somente o acompanhamento clínico. $\mathrm{Na}$ avaliação da diferença entre o $\Delta \mathrm{Po}$ nos três grupos, na primeira hora, foi encontrada diferença estatisticamente signi- 


\section{ANÚNCIO}




\section{ANÚNCIO}




\begin{tabular}{|c|c|c|c|c|c|c|c|c|c|}
\hline $\begin{array}{l}\text { Po pré } \\
\text { Po pré } \\
\text { Po pré }\end{array}$ & $\begin{array}{l}\text { A } \\
B \\
C\end{array}$ & $\begin{array}{l}14 \\
11 \\
11\end{array}$ & $\begin{array}{l}11,71 \\
11,82 \\
12,18\end{array}$ & $\begin{array}{l}4,73 \\
3,43 \\
4,79\end{array}$ & $\begin{array}{c}19,0 \\
18,00 \\
20,0\end{array}$ & $\begin{array}{c}1,000 \\
8,00 \\
4,00\end{array}$ & $\begin{array}{c}12,50 \\
10,000 \\
11,00\end{array}$ & $\begin{array}{c}9,000 \\
9,25 \\
9,250\end{array}$ & $\begin{array}{l}15,00 \\
14,75 \\
16,00\end{array}$ \\
\hline $\begin{array}{l}\text { Po 1h } \\
\text { Po 1h } \\
\text { Po 1h }\end{array}$ & $\begin{array}{l}A \\
B \\
C\end{array}$ & $\begin{array}{l}14 \\
11 \\
11\end{array}$ & $\begin{array}{l}13,29 \\
10,09 \\
15,27\end{array}$ & $\begin{array}{l}4,63 \\
3,14 \\
7,99\end{array}$ & $\begin{array}{c}23,0 \\
15,00 \\
34,0\end{array}$ & $\begin{array}{c}5,000 \\
4,00 \\
8,00\end{array}$ & $\begin{array}{c}11,50 \\
10,000 \\
12,00\end{array}$ & $\begin{array}{c}10,000 \\
10,00 \\
10,250\end{array}$ & $\begin{array}{l}17,00 \\
12,00 \\
18,50\end{array}$ \\
\hline $\begin{array}{l}\text { Po 4h } \\
\text { Po 4h } \\
\text { Po 4h }\end{array}$ & $\begin{array}{l}A \\
B \\
C\end{array}$ & $\begin{array}{l}14 \\
11 \\
11\end{array}$ & $\begin{array}{l}12,79 \\
10,36 \\
15,45\end{array}$ & $\begin{array}{l}4,42 \\
2,80 \\
7,94\end{array}$ & $\begin{array}{c}24,0 \\
15,00 \\
36,0\end{array}$ & $\begin{array}{c}7,000 \\
6,00 \\
8,00\end{array}$ & $\begin{array}{c}11,00 \\
10,000 \\
12,00\end{array}$ & $\begin{array}{c}10,000 \\
9,00 \\
10,250\end{array}$ & $\begin{array}{l}16,00 \\
11,00 \\
17,50\end{array}$ \\
\hline $\begin{array}{l}\text { Delta } 4 \mathrm{~h} \\
\text { Delta } 4 \mathrm{~h} \\
\text { Delta } 4 \mathrm{~h}\end{array}$ & $\begin{array}{l}A \\
B \\
C\end{array}$ & $\begin{array}{l}14 \\
11 \\
11\end{array}$ & $\begin{array}{c}1,07 \\
-1,45 \\
3,27\end{array}$ & $\begin{array}{l}5,25 \\
3,47 \\
6,48\end{array}$ & $\begin{array}{l}11,0 \\
5,00 \\
20,0\end{array}$ & $\begin{array}{c}-9,000 \\
-7,00 \\
-2,00\end{array}$ & $\begin{array}{c}1,00 \\
-1,000 \\
2,00\end{array}$ & $\begin{array}{c}-2,000 \\
-4,50 \\
-0,750\end{array}$ & $\begin{array}{l}3,00 \\
0,75 \\
5,00\end{array}$ \\
\hline
\end{tabular}

ficativa, sendo que o grupo B apresentou variação significativamente maior que o grupo C. O grupo A não diferiu estatisticamente dos outros dois grupos. Já na quarta hora, esta diferença não foi encontrada (Tabela 2).

As variações dentro de cada grupo não foram grandes o suficiente para suscitar diferença estatisticamente significativa em nenhum dos intervalos (Grupo A: $p=0,08140$; Grupo B: $p=0,1021$, Grupo $\mathrm{C}: \mathrm{p}=0,3379)$.

Não foi encontrada correlação forte e significativa entre energia e os deltas nos dois intervalos em nenhum grupo (Tabela 3).

\section{DISCUSSÃO}

A freqüência de hipertensão ocular após capsulotomia posterior com YAG laser em grupos não tratados com drogas anti-hipertensivas varia muito de estudo para estudo ${ }^{1,2,4-13,18}$.

Neste estudo apenas dois pacientes do grupo controle apresentaram hipertensão intra-ocular. Estes valores estão abaixo dos encontrados por Silverstone e col. ${ }^{9}$ (Controle: 39\%, apraclonidina: 7\%) e por Cullom e col. ${ }^{12}$ (Controle: 59\%, apraclonidina: 13\%). Esta diferença pode ser explicada pelo fato de ter sido excluído glaucomatosos desse estudo, o que não ocorreu nos trabalhos acima citados. Segundo Shani e col. ${ }^{5}$ e Mello \& Prata ${ }^{15}$, os glaucomatosos apresentam maior risco de aumento da Po pós capsulotomia com YAG laser.

Não foi encontrada diferença estatisticamente significativa entre as Po dos 3 grupos em nenhum dos intervalos analisados. No entanto, somente o grupo controle apresentou casos de hipertensão ocular $(18,2 \%)$. Individualmente o maior aumento de pressão ocorreu no grupo controle (aumento de 18 $\mathrm{mmHg}$ ), sendo que neste grupo o aumento da Po era notado na

\begin{tabular}{|c|c|c|c|c|c|c|c|}
\hline Período & Grupo & Comparação & $\mathbf{N}$ & Média & Desvio Padrao & $\mathbf{F}$ & $\mathbf{P}$ \\
\hline \multirow[t]{6}{*}{$1^{\circ}$ hora } & A & & 14 & 7,87 & 33,0 & & \\
\hline & B & & 11 & $-27,05$ & 45,2 & & \\
\hline & C & & 11 & 14,29 & 28,4 & 4,31 & $0,021^{*}$ \\
\hline & & $B$ vs $A$ & & & & & $>0,05^{\star *}$ \\
\hline & & $B$ vs $C$ & & & & & $<0,05^{\star *}$ \\
\hline & & $C$ vs $A$ & & & & & $>0,05^{\star *}$ \\
\hline \multirow[t]{3}{*}{$4^{\circ} \stackrel{\text { hora }}{ }$} & $A$ & & 14 & 9,13 & 41,0 & & \\
\hline & B & & 11 & $-17,84$ & 34,1 & & \\
\hline & $\mathrm{C}$ & & 11 & 17,42 & 32,2 & 2,85 & $0,072^{*}$ \\
\hline
\end{tabular}




\begin{tabular}{|lccc|}
\hline \multicolumn{2}{|c|}{ Tabela 3. Correlação entre energia e os Deltas $(\Delta)$ da $1^{\text {a.. }}$ e $4^{\text {a }}$. } \\
hora \\
Grupo & Período & r & p \\
A & $1^{\text {a }}$ hora & 0,0915 & 0,8083 \\
& $4^{\underline{a}}$ hora & $-0,0552$ & 0,8438 \\
B & $1^{\text {a }}$ hora & 0,0915 & 0,7755 \\
& $4^{\underline{a}}$ hora & 0,446 & 0,159 \\
C & $1^{\text {a }}$ hora & 0,248 & 0,176 \\
& $4^{\text {a }}$ hora & 0,450 & 0,595 \\
\hline
\end{tabular}

$1^{a}$ hora de pós-operatório e aumentava na $4^{\mathrm{a}}$ hora. A elevação da Po no grupo controle tendeu a prolongar-se, estando de acordo com os achados de Brown e col. ${ }^{11} \mathrm{e}$ de Minello e col. ${ }^{18}$.

A apraclonidina $1 \%$ foi a droga que levou a uma maior redução da Po na $1^{\mathrm{a}}$ hora após o laser. No entanto, não houve diferença estatisticamente significativa em relação ao grupo do latanoprost no mesmo horário. $\mathrm{Na} 4^{a}$ hora os três grupos foram estatisticamente semelhantes. Sendo assim, os resultados deste estudo estão de acordo com os de Silverstone e col. ${ }^{9}$, Pollack e col. ${ }^{10}$, Brown e col. ${ }^{11}$, Cullom e col. ${ }^{12}$, Prata Jr. e col. ${ }^{13}$ que usaram apraclonidina $1 \%$ e chegaram à conclusão de que esta droga é eficaz na prevenção da hipertensão ocular pós YAG laser.

Desconhecemos trabalhos publicados sobre o uso de latanoprost na prevenção da hipertensão ocular após capsulotomia posterior com YAG Laser.

A quantidade de energia utilizada durante o laser tem sido considerada por alguns autores uma das causas de aumento da pressão após o procedimento ${ }^{3,4}$. Esta correlação não foi encontrada em outros estudos ${ }^{1,5,6}$, assim como neste.

Embora a apraclonidina tenha sido mais eficaz que o latanoprost para a profilaxia da hipertensão ocular póscapsulotomia com YAG-Laser, a diferença não alcançou significância estatística, o que pode ter ocorrido devido ao tamanho da amostra. Porém, quando comparada ao grupo controle, o grupo da apraclonidina apresentou melhores e significativos resultados em relação ao grupo do latanoprost.

Os autores concluem pela maior eficácia do uso de apraclonidina $1 \%$ em pacientes normais quando da profilaxia da hipertensão pos-capsulotomia com YAG-Laser.

\section{SUMMARY}

Purpose: To compare the efficacy of $0.005 \%$ latanoprost and $1 \%$ apraclonidine in the prevention of an increase of intraocular pressure (IOP) after Nd: YAG laser capsulotomy in nonglaucomatous patients.

Methods: Thirty-six patients were included and divided in to three groups. Patients received $0.005 \%$ latanoprost. $1 \%$ apraclonidine or placebo. Four measurements of IOP were made: before instillation of any drug, 30 minutes after cycloplegia, 1 hour and 4 hours after laser.

Results: No significant difference was found between the three groups regarding age, initial intraocular pressure, pressure 30 minutes after cycloplegia and amount of laser energy used. In the apraclonidine group, at the first hour after laser, both the absolute and percentual intraocular pressure changes, were statistically lower than those of the control group $(p<0.05)$. The latanoprost group had was not significantly different from the other groups $(p>0.05)$. The IOP of the 3 groups was statistically similar at the fourth hour after the laser $(p>0.05)$. There were no differences in the measurements of intraocular pressure between the four intervals. The amount of laser energy used during the procedure had no correlation with IOP rise ( $p>0.05)$.

Conclusion: The authors conclude that $1 \%$ apraclonidine is more effective to control the IOP rise after YAG laser capsulotomy when compared to $0.005 \%$ latanoprost .

Keywords: Glaucoma: capsulotomy; ocular hipertension, apraclonidine, latanoprost prevention.

\section{REFERÊNCIAS BIBLIOGRÁFICAS}

1. Slomovic AR, Parrish II RK. Acute elevations of intraocular pressure following Nd: YAG laser posterior capsulotomy. Ophthalmology 1985;92(7): 973-6.

2. Ladas ID, Pavlopoulos GP, Kokolakis SN, Theodossiadis GP. Prophylatic use of acetazolamide to prevent intraocular pressure elevation following Nd-YAG laser posterior capsulotomy. Br J Ophthalmol 1993;77:136-8.

3. Wetzel W. Ocular aquous humor dynamics after photodiruptive laser surgery procedures. Ophthalmic Surgery 1994;25(5):298-302.

4. Channel MM, Beckman H. Intraocular pressure changes after Neodymium YAG laser posterior capsulotomy. Arch Ophtalmol 1984;102:1024-6.

5. Shani L, David R, Tessler L, Rosen S, Schneck M. Intraocular pressure after neodymium: YAG laser treatments in the anterior segment. J Cataract Refractive Surg 1994;20:455-8.

6. Paranhos FRL, Ribeiro RM, Paranhos Jr. A. Profilaxia eficaz da hipertensão pós YAG - Laser para capsulotomia posterior. Rev Bras Oftal 1995;54(8):33-9.

7. Arieta CEL, Barbosa KB, Rocha EM, Castro RS, José NK. Pilocarpina a $2 \%$ na prevenção da elevação da pressão intra-ocular pela aplicação de Neodymium YAG laser em capsulotomia posterior. Arq Bras Oftal 1993;56(5):266-8.

8. Stilma JS, Boen-tan TN. Timolol and intraocular pressure elevation following Neodymium: YAG laser surgery. Doc Ophtalmologica 1986;61:233-9.

9. Silverstone DE, Brint SF, Olander KW, Taylor RB, McCarty GR, deFaller JM. Prophylatic use of apraclonidine for intraocular pressure increase after Nd:YAG capsulotomies. Amer J Ophthal 1992;113:401-5.

10. Pollack IP, Brown RH, Crandall AS, Robin AL, Stewart RH, White GL. Prevention of the rise in intraocular pressure following Nd-YAG posterior capsulotomy using topical 1\% apraclonidine. Arch Ophthalmol 1988;106:756-7.

11. Brown RH, Stewart RH, Lynch MG, Crandall AS, Mandell AI, Wilensky JT, Schwartz AL, Gaasterland DE, DeFaller JM, Higginbotham EJ. ALO 2145 Reduces the intraocular pressure elevation after anterior segment laser surgery. Ophthalmology 1988;95(3):378-83.

12. Cullom RD, Schwartz LW. The effect of apraclonidine on the intraocular pressure of glaucoma patients following $\mathrm{Nd}$ laser posterior capsulotomy. Ophthalmic surgery 1993;24(9):623-6.

13. Prata Jr JA, Rehder JRCL, Mello PAA. Apraclonidine and early postoperative intraocular hypertension after cataract extraction. Acta Ophthalmol 1992; 70:434-9.

14. Hoyng PFJ, Rulo A, Greve E, Watson P, Alm A. The additive intraocular pressure-lowering effect of latanoprost in combined therapy with other ocular hypotensive agents. Surv Ophtalmol 1997;41(Supl.2):S93-8.

15. Mello PAA, Prata Jr JA. Capsulotomia posterior com Nd:YAG laser em glaucomatosos pseudofácicos. Arq Bras Oftal 1993;56(6):315-9.

16. Gharagozloo NZ, Relf SL, Brubaker RF. Aqueous flow is reduced by the alpha adrenergic agonist, apraclonidine hydrochloride (ALO 2145). Ophtalmology 1988;95:1217.

17. Toris CB, Camras CB, Yablonski ME, Brubaker RF. Effects of exogenous prostaglandins on aqueous humor dynamics and blood-aqueous barrier fuction. Surv of Ophthalmol 1997;41(Supl.2):569-75.

18. Minello AAP, Prata Jr. JA, Abujamra S, Mello PAA. Pressão intra-ocular após capsulotomia posterior com laser de Neodymium Yag em olhos pré-tratados com dorzolamida. Arq Bras Oftal 1998;6(2):160-4. 


\section{ECO - Educação Continuada em Oftalmologia 0 Conselho Brasileiro de Oftalmologia, com patrocínio de Alcon, Allergan-Frumtost e Varilux

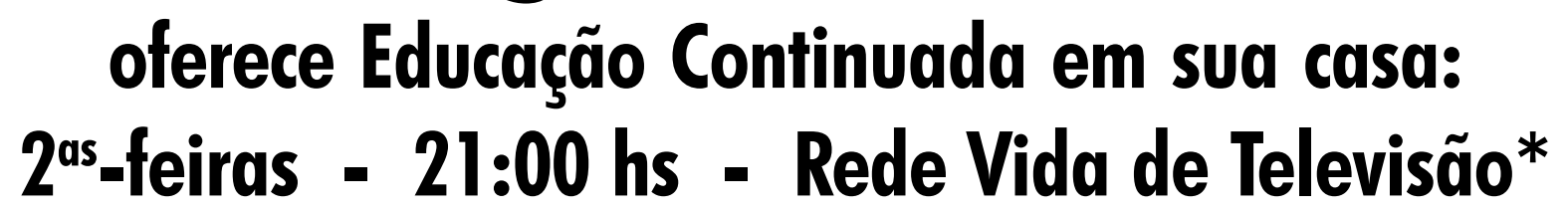

Desde setembro de 1996 o Conselho Brasileiro de Oftalmologia vem realizando uma antiga aspiração: manter uma programação permanente de ECO - Educação Médica Continuada em Oftalmologia. A ECO é transmitida às $2^{a}$ s e $4^{a}$ s segundas-feiras do mês, às 21 h00 pela Rede Vida de Televisão*.

Em seus primeiros 18 meses a programação da ECO abordou temas gerais de oftalmologia. Desde maio de 1998 os programas tem novo formato: uma Aula de 10 minutos, seguida de Mesa Redonda com dois debatedores. Cada quatro programas correspondem a uma fita (vídeo) ${ }^{* *}$ com duas horas de conhecimentos científicos, transmitidos por oftalmologistas selecionados.

\begin{tabular}{|c|c|c|c|}
\hline MÓDULO & TÍTULO & PALESTRANTES & $\begin{array}{l}\text { DATA } \\
\text { Exibição }\end{array}$ \\
\hline MÓDULO & $17^{\circ}$ & & \\
\hline 78 & $\begin{array}{l}\text { Çorreção Cirúrgica da Alta Miopia } \\
\text { Wallace Chamon }\end{array}$ & $\begin{array}{l}\text { Palestrante: Norma Allemann } \\
\text { Discutidores: Fernando Trindade / Walton Nosé }\end{array}$ & $11 / 10 / 99$ \\
\hline 79 & $\begin{array}{l}\text { Degeneração Macular Relacionada a Idade } \\
\text { Mário Luiz R. Monteiro }\end{array}$ & $\begin{array}{l}\text { Palestrante: Marcio Bittar Nehemy } \\
\text { Discutidores: Jorge Mitre / Michel Eid Farah }\end{array}$ & $25 / 10 / 99$ \\
\hline 80 & $\begin{array}{l}\text { Refratometria na Era do Laser } \\
\text { Hamilton Moreira }\end{array}$ & $\begin{array}{l}\text { Palestrante: Helena Tanaka } \\
\text { Discutidores: Wallace Chamon / Aderbal A. Alves }\end{array}$ & $08 / 11 / 99$ \\
\hline 81 & $\begin{array}{l}\text { Membrana Amniótica } \\
\text { Wallace Chamon }\end{array}$ & $\begin{array}{l}\text { Palestrante: José Álvaro Pereira Gomes } \\
\text { Discutidores: Hélia Angotti / Amélia Kamegasawa }\end{array}$ & $22 / 11 / 99$ \\
\hline MÓDULO & $15^{\circ}$ & & \\
\hline 82 & $\begin{array}{l}\text { Marketing e Ética em Offtalmologia } \\
\text { Wallace Chamon }\end{array}$ & $\begin{array}{l}\text { Palestrante: Pedro Paulo R. Monteleone } \\
\text { Discutidores: Claudio Lottenberg / Marcelo Ventura }\end{array}$ & $13 / 12 / 99$ \\
\hline 83 & $\begin{array}{l}\text { Retinopatia Diabética } \\
\text { Mário Luiz Ribeiro Monteiro }\end{array}$ & $\begin{array}{l}\text { Palestrante: Jacó Lavinsky } \\
\text { Discutidores: Suel Abujamra / João } 0 \text {. R. Gonçalves }\end{array}$ & $27 / 12 / 99$ \\
\hline 84 & $\begin{array}{l}\text { Ceratocone } \\
\text { Wallace Chamon }\end{array}$ & $\begin{array}{l}\text { Palestrante: Marcelo Cunha } \\
\text { Discutidores: Sérgio Kwitko / Saly Moreira }\end{array}$ & $10 / 01 / 00$ \\
\hline 85 & $\begin{array}{l}\text { Transplante e Doação de Córnea } \\
\text { Wallace Chamon }\end{array}$ & $\begin{array}{l}\text { Palestrante: Élcio Hideo Sato } \\
\text { Discutidores: Hamilton Moreira / José A. A. Milani }\end{array}$ & $24 / 01 / 00$ \\
\hline
\end{tabular}

* A Rede Vida informa como sintonizá-la em todo país: (OXX17) 224-5556 falar com Daniel. Regulagem da parabólica: canal 5 A 2 Polarização Horizontal Freqüência: $3.890 \mathrm{mHz}$

** Os vídeos estão a venda por RS 20,00 + despesas postais. Maiores informações: CBO - Tel.: (0XX11) 3266-4000 - Fax: 3171-0953 